\title{
Cycles of growth and destruction
}

\section{Recent advances in understanding of the eukaryotic cell cycle and its disruption in cancer have been dramatic. Participants at Nature's conference "Cycling out of control" (Paris, 1-2 December) found that they continue.}

READERS of Nature will have noted several papers about the ecology of Scandinavian voles over the past few years. These rodents undergo marked swings in population, reproducing at very high rates in good years, only to suffer catastrophic losses as their food supply runs out and their predators multiply. While the details of this process have puzzled theoretical ecologists for some time, it is clear that in an ecosystem, at least, unfettered reproduction has its limits.

Within a multicellular organism, things are not so simple. In this case, a cell whose replication proceeds unchecked will shortly kill the organism from which it sprang, along with itself. The very behaviour characteristic of all organisms from bacteria to blue whales, to multiply as fast as one's surroundings will permit, is here disastrous. So the cells of metazoan animals have grafted a stringent set of checkpoints onto the cycle that directs eukaryotic cell division.

Of course, that cycle is itself carefully controlled, so that a cell that has replicated its DNA cannot do so again before mitosis, and a cell that has not cannot undergo mitosis. In fission yeast, the latter block is imposed by cdc 18 , a protein made late in $\mathrm{G}_{1}$ which is essential for DNA replication and prevents mitosis until it is complete (Paul Nurse, ICRF, London, whose epic performance when faced with a slide projector which obstinately refused to recognize his slides will not soon be forgotten by anyone present). But what prevents mitosis before then? The answer appears to be a protein made early in $\mathrm{G}_{1}$ called rum1, which is a potent inhibitor of mitotic cyclins. These cyclins themselves have the opposite role: they are made only in $\mathrm{G}_{2}$, and prevent replication unless preceded by mitosis.

More details of the process have emerged from studies of budding yeast, where the activity of mitotic-type cyclins called Clb5 and 6 (which are, confusingly, necessary for DNA replication) appears to be held in check by a protein called $\mathrm{p} 40^{\mathrm{Sicl}}$ (Kim Nasmyth, Institute of Molecular Pathology, Vienna). The latter is earmarked for destruction at the appropriate moment by a ubiquitin-conjugating enzyme encoded by the $C d c 24$ gene, but only after $\mathrm{Cdc} 6$ (the budding yeast equivalent of cdc18) has triggered the assembly of replication complexes. In Drosophila and Xenopus embryos, these controls appear to be added progressively during development, as the initial rapid cycles are slowed by the introduction of refinements such as $\mathrm{G}_{1}$ and $\mathrm{G}_{2}$ (Pat O'Farrell, University of California, San Francisco, and
Tim Hunt, ICRF, South Mimms, England).

Onto this base, metazoan cells add a programme that directs them to die rather than proliferate when damaged or in response to an inadequate signal. This pathway can be blocked by the protein bcl- 2 . But whereas there is apparently only one such protein in Caenorhabditis elegans, it has numerous relatives in mammalian cells, all of which can form heterodimers with at least one other family member, and some of which actually promote apoptosis (Stan Korsmeyer, Washington University, St Louis). Some viral proteins have similar properties. The $19 \mathrm{kD}$ E1B protein of adenovirus may even improve upon bcl-2 by protecting the lamins of the nuclear envelope, which are broken down in apoptosis (Eileen White, Rutgers University).

But perhaps the most important protein inactivated in cancer is p53, which directs cell-cycle arrest or apoptosis in response to DNA damage. The E6 gene of oncogenic human papilloma viruses, for instance, inactivates p53, increasing the frequency of DNA rearrangements by up to five orders of magnitude, without changing cellular morphology or doubling time (Thea Tlsty, University of North Carolina).

Exactly how DNA damage activates p53 is still unclear. In budding yeast, damage is apparently detected by DNA polymerase $\epsilon$, which signals through the kinases Sad1 and Dun 1 to initiate the repair process (Steve Elledge, Baylor College of Medicine, Houston). But however the process works in metazoans, p53 is the commonest gene lost in frank neoplasia, and its absence markedly accelerates gross chromosomal rearrangement (Andrew Wyllie, University of Edinburgh). Cells in this condition are also very resistant to ionizing radiation and cytotoxic drugs, and the discovery that taxol ${ }^{*}$, at least, can induce apoptosis by a route independent of p53 is therefore particularly welcome (Scott Lowe, MIT).

Other systems may also be inactivated in the majority of cancers. The retinoblastoma protein, Prb, sequesters the transcription factor E2F (needed for progress through $S$ phase) until it is inactivated by a complex of cyclin D and cdk4 or 6 . This in turn can be inactivated by the small inhibitors p 15 or p16, effectively blocking the cell cycle. Many oncogenic viruses encode $\mathrm{pRb}$ inhibitors, and up to 40 per cent of tumours have no functional copy of the gene. But it is now

Nature has been informed by Bristol-Myers Squibb that "taxol" has been a trade name registered in that company's name since 1992. clear that in other malignancies, there are defects elsewhere in the pathway, including (in at least one case of familial melanoma) the gene for p16 (Ed Harlow, Massachusetts General Hospital, Boston).

The importance to an emergent cancer of preventing apoptosis is underlined by the phenotype of mice lacking the ras GTPaseactivating protein GAP. So far from promoting cancer by activating ras, the knockout is in fact lethal in utero, causing massive apoptosis throughout the embryo (Tony Pawson, Samuel Lundenfeld Research Institute, Mount Sinai Hospital, Toronto). Other ras targets may control cellular behaviour, influencing membrane ruffling and the activity of the related GTPase ral (John Cooper, Fred Hutchinson Cancer Research Center, Seattle).

The extracellular signals transmitted by ras eventually trigger transcription, but the pattern can be unexpectedly complex. Expression of the proto-oncogene fos, for instance, appears to depend on the concerted action of four transcription factors, all of which are necessary in vivo (Tom Curran, Roche Institute of Molecular Biology, Nutley, New Jersey). Even the response to a simple rise in cAMP levels depends on a balance between stimulatory and inhibitory forms of the transcription factor CREM, which in hamsters directs rhythmic behaviour in addition to its role in the cell cycle (Paolo Sassone-Corsi, CNRS, Strasbourg).

Only seldom is the link between an extracellular signal and the cell cycle obvious. TGF $\beta$ is an honourable exception, as it appears to block cycling by inducing transcription of the cdk inhibitor p15 (David Beach, Cold Spring Harbor Laboratory, New York). But Beach also presented experiments which indicate that the kinase raf, once activated by ras, can activate some forms of $\operatorname{cdc} 25$, itself a major activator of cyclin/cdk complexes. In some cases, therefore, the route from signal to cycle may be more direct than expected.

Such a remarkable result immediately suggests a new set of genes to screen for oncogenic alterations in cancer biopsies. Even before this is done, however, it is clear that recent advances in our understanding of the cell cycle have revolutionized our view of cancer. Loss of proper checkpoints, allowing resistance to apoptotic signals and an increased rate of mutation, can be seen as a hallmark of the condition. It will be exciting to watch as this and future advances are converted into improvements in patient care.

Nicholas Short 